\title{
STUDIES IN THE REPRESENTATION THEORY OF FINITE SEMIGROUPS
}

\author{
BY \\ YECHEZKEL ZALCSTEIN( $\left.{ }^{1}\right)$
}

\begin{abstract}
This paper is a continuation of [14], developing the representation theory of finite semigroups further. The main result, Theorem 1.24, states that if the group of units $U$ of a mapping semigroup $(X, S)$ is multiply transitive with a sufficiently high degree of transitivity, then for certain irreducible characters $x$ of $U, x$ can be "extended" formally to an irreducible character of $S$. This yields a partial generalization of a well-known theorem of Frobenius on the characters of multiply-transitive groups and provides the first nontrivial explicit formula for an irreducible character of a finite semigroup. The paper also contains preliminary results on the "spectrum" (i.e., the set of ranks of the various elements) of a mapping semigroup.
\end{abstract}

Introduction. The well-known results of Clifford-Suschkewitch and of Munn [4, Chapter 5], [14, Section 2] reduce, in principle, the problem of finding the irreducible representations of a finite semigroup to that of finding the representations of its maximal subgroups. However, these results are not in readily usable form. For example, the problem of finding the radical of a semigroup algebra has been solved only recently, and only for the special case of a 0 -simple semigroup [14, (2.9) and (2.11)]. Most recent research in the field has been concerned with extending Munn's results to infinite semigroups.

This paper is an attempt to obtain sharper results for finite semigroups. In particular, we generalize some well-known results about the characters of multiplytransitive permutation groups (1.16-1.34). The main results, Theorems 1.24 and 1.41 , state that if the group of units $U$ of a mapping semigroup $(X, S)$ is multiplytransitive with a sufficiently high degree of transitivity, then for certain irreducible characters $\chi$ of $U, \chi$ can be "extended" formally to an irreducible character of $S$. This yields a partial generalization of a well-known theorem of Frobenius on the characters of multiply transitive permutation groups and provides the first nontrivial explicit formula for an irreducible character of a finite semigroup. The paper also contains some preliminary results on the "spectrum" of a mapping semigroup.

We hope that as the character theory of finite semigroups develops, it will have

Received by the editors December 14, 1970.

AMS 1969 subject classifications. Primary 2090; Secondary 2092.

Key words and phrases. Finite semigroup, irreducible representation, character, $k$-transitive.

(1) This research was supported in part by the U.S. Army Research Office (Durham) under contract DA-31-124-ARO-D-394.

Copyright (C) 1971, American Mathematical Society 
significant applications to problems of structure, as has been the case in group theory.

This paper is a revised version of Chapter 4 of the author's Ph.D. thesis [18]. The remainder of the thesis, dealing with the group-complexity of finite semigroups, will appear elsewhere.

1. Representation theory. We assume that the reader is familiar with the algebraic theory of finite semigroups [4], [8] as well as the elementary representation theory of semigroups [4, Chapter 5], [14].

1.1. Notation. Throughout this paper, $S$ and $T$ with various subscripts will denote finite semigroups and $G, H$ and $U$ with various subscripts will denote finite groups. $\mathscr{S}_{n}$ denotes the symmetric group on $n$ symbols. $X=D_{n}=\{1,2, \ldots, n\}$, and $\left(X, \mathscr{S}_{n}\right)$ denotes $\mathscr{S}_{n}$ acting in the natural way on $X$.

If $J$ is a regular $\mathscr{J}$-class of $S, G_{J}$ denotes a maximal subgroup of $S$ contained in $J$ and $C_{J}$ the structure matrix of the 0 -simple semigroup $J^{0}$.

$K$ denotes an algebraically closed field of characteristic zero. All representations considered are $K$-representations, unless stated otherwise. $Z$ denotes the ring of integers and $Q$ its quotient field. $\mathscr{C}$ denotes the field of complex numbers. $\varphi, \psi$ and $\theta$ with various subscripts denote representations and $V, W$ with various subscripts denote right $K[S]$-modules. $1_{S}$ denotes the principal character on $S$ and if there is no danger of confusion we will denote it simply by 1 .

Let $\varphi$ be a representation of $S$ and let $G$ be a maximal subgroup of $S$, then $\varphi_{G}$ denotes the group representation of $G$ derived from $\left.\varphi\right|_{G}$ by discarding null constituents. If $\chi$ is a character of $S$, then $\varphi(\chi)$ denotes any representation with character $\chi$.

All $K[S]$-modules considered are right modules. When there is no danger of confusion we will identify an irreducible representation with its character. If $\varphi$ and $\psi$ are representations, $\varphi \leqq \psi$ means that every nonnull constituent of $\varphi$ is a constituent of $\psi$. The same notation is used for characters. If $\varphi$ is a nonnull irreducible representation and $\psi$ is an arbitrary representation of $S, \#(\varphi, \psi)$ is the number of constituents of $\psi$ which are similar to $\varphi$. If $V$ and $W$ are the representation modules of $\varphi$ and $\psi$, respectively, then $\#(V, W)=\#(\varphi, \psi)$.

All undefined notation follows [8] and [14].

A famous theorem of Burnside [5, (36.1)] states that if a group has a faithful finite-dimensional complex representation and there is an upper bound $m$ on the orders of all elements in the group then the group is finite.

This has a partial generalization as follows:

1.2. Proposition. Let $S$ be a (possibly infinite) semigroup such that, for each $s \in S,\langle s\rangle$ is finite and there exists a positive integer $m$ such that the periods of all elements of $S$ are bounded by $m$. If $S$ has a faithful finite-dimensional irreducible $\mathscr{C}$-representation, then $S$ is finite.

Proof. The proof follows by trivial modifications of the proof of Burnside's theorem in [5]. Let $s \in S$ and let $r$ and $p$ be the index and period of $s$, respectively 
[8, p. 11]. Then $s^{r+p}=s^{r}$, thus if $\varphi$ is an $n$-dimensional representation of $S$, $\varphi(s)^{r}\left[\varphi(s)^{p}-1\right]=0$ and hence the eigenvalues of $\varphi(s)$ are 0 or $p$ th roots of unity. By hypothesis, the periods of all the elements are bounded by $m$ and thus for all $s \in S$ the eigenvalues of $\varphi(s)$ are 0 or $k$ th roots of unity, for some $k \leqq m$. Let $\chi=\chi(\varphi)$, then, for all $s \in S, \chi(s)$ is a sum of at most $n k$ th roots of unity and thus the set $\{\chi(s): s \in S\}$ is finite.

Now, assume that $\varphi$ is faithful and irreducible. Then by the Frobenius-Schur theorem [14, (1.20)] there exist $n^{2}$ elements $s_{1}, s_{2}, \ldots, s_{n^{2}} \in S$ such that $\left\{\varphi^{i j}\left(s_{k}\right): 1 \leqq i, j \leqq n, 1 \leqq k \leqq n^{2}\right\}$ are linearly independent over $K$. For $s \in S$,

$$
\chi\left(s_{k} s\right)=\sum_{i=1}^{n} \varphi^{i i}\left(s_{k} s\right)=\sum_{i, j=1}^{n} \varphi^{i j}\left(s_{k}\right) \varphi^{j i}(s)
$$

The right-hand side is a set of $n^{2}$ linear equations in the $n^{2}$ unknowns $\varphi^{i j}(s)$, and the rows of the matrix of coefficients being linearly independent, there is a unique solution for the $\varphi^{i j}(s)$. But $\chi$ takes on only a finite number of possible values, and hence so does each $\left\{\varphi^{i j}(s): s \in S\right\}$. Thus $\varphi(S)$ and hence $S$ is finite.

1.3. Remark. Unlike the group case, the conclusion of Proposition 1.2 does not hold if $\varphi$ is reducible even if the bound $m$ is 1 as is shown by the following simple example.

Let $S$ be the semigroup of all $n \times n$ lower strictly triangular matrices over $\mathscr{C}$. Every element of $S$ is nilpotent and thus has period 1 . The identity map is a faithful $n$-dimensional representation. Thus all the hypotheses of 1.2 hold, except the irreducibility of the representation. However, $S$ is infinite.

The next result is a generalization of a theorem of Schur which states that a finitely generated torsion group having a faithful finite-dimensional representation is finite $[5,(36.2)]$.

1.4. Proposition. Let $S$ be a finitely generated torsion semigroup. If $S$ has a faithful finite-dimensional irreducible $\mathscr{C}$-representation $\varphi$, then $S$ is finite.

Proof. As in 1.2, the proof is a slight modification of the standard proof of Schur's theorem. The idea of the proof is to get a uniform bound on the periods of all elements of $S$ and then apply 1.2. Let $s_{1}, s_{2}, \ldots, s_{n}$ be a set of generators for $S$. Let $E$ be the field obtained by adjoining to $Q$ the entries of all the matrices $\varphi\left(s_{i}\right)$, $1 \leqq i \leqq n$. Thus each eigenvalue of $\varphi(s)$ is 0 or a root of unity which satisfies an equation of degree $l=\operatorname{deg}(\varphi)$ with coefficients in $E$. As in the proof of Schur's theorem (see [5, pp. 252-254]) there are finitely many such roots of unity, thus there exists a positive integer $m$ such that each eigenvalue of $\varphi(s)$ is 0 or an $m$ th root of unity. Then $S$ is finite by the proof of 1.2.

1.5. FACT. Let $F$ be a subfield of $K$. Let $J$ be a regular $\mathscr{J}$-class of $S$, $J^{0} \simeq \mathscr{M}^{0}(G, A, B, C)$ and let $\varphi \in \operatorname{IRR}(G)$. If $\varphi$ is similar to an F-representation, then $\varphi^{\bar{\pi}}$ is similar to an F-representation. 
Proof. Replace " $K_{0}[S]$ " by " $F_{0}[S]$ " in (2.9) of [14]; the proof goes through except that we have possibly fewer irreducible $F$-representations than irreducible $K$-representations of $G$. Furthermore, $\varphi(C)$ is a matrix over $F$, and thus the matrices $A$ and $B$ in the proof of $[14,(2.7)]$ are over $F$. Thus $\varphi^{\wedge}$ constructed in [14, (2.11)] is an $F$-representation. Finally, the proof of $[14,(2.2)]$ requires finding $x \in K[J \cup F(J)]$ such that $\varphi^{\sim}(x)=I_{n}$. Since this involves only solving linear equations and $\varphi^{\approx}$ is an $F$-representation, the coefficients of $x$ are from $F$ and thus for all $s \in S$ the coefficients of $x s$ are from $F$. Thus $\varphi^{\bar{\wedge}}$ is an $F$-representation.

1.6. Corollary. All irreducible representations of $F_{R}(X)$ are similar to $Q$ representations.

Proof. As is well known (see e.g., [5]), every irreducible representation of $\mathscr{S}_{k}$ is similar to a $Q$-representation and since every maximal subgroup of $F_{R}(X)$ is isomorphic to $\mathscr{S}_{k}$, for some $k$, the corollary follows from Fact 1.5.

1.7. FACT. Every $Q$-representation of $S$ is similar to a Z-representation (i.e., to a representation by matrices over $Z$ ).

Proof. The standard proof of the corresponding theorem for groups (see e.g., $[6,(4.1)])$ goes over without change.

1.8. REMARK. It follows from 1.5 and 1.7 that if $G$ is a maximal subgroup of $S$, then $1_{G}^{\widehat{\widehat{G}}}$ is similar to a $Z$-representation, thus in particular $\chi(1 \overline{\widehat{G}})$ is $Z$-valued. Further, by Fact 3.13 of [14], the characters of a combinatorial semigroup have nonnegative integer values. Thus it has been conjectured that the values of $\chi(1 \overline{\widehat{G}})$ are nonnegative integers. Let $C$ be the structure matrix of the $\mathscr{J}$-class of $S$ containing $G$. The assertion is trivially true if the rows of $1_{G}^{*}(C)$ are linearly independent since then by (2.16) of [14], $1_{G} \overline{\widehat{G}}=1_{G}^{*}\left(R M_{J}\right)$. However, it is false in general, as shown by the following example.

Let $S$ be a chain of $3 \mathscr{J}$-classes $\{0\}=J_{0}<J_{1}<J_{2}$ with $J_{2} \simeq Z_{2}$ and $J_{1}$ $\simeq M^{0}(\{1\},\{1,2,3,4\},\{1,2,3,4\}, C)$,

$$
C=\left[\begin{array}{llll}
1 & 1 & 0 & 0 \\
0 & 0 & 1 & 1 \\
1 & 0 & 1 & 0 \\
0 & 1 & 0 & 1
\end{array}\right]
$$

and for the generator $g$ of $J_{2}$,

$$
R M_{J_{1}}(g)=\left[\begin{array}{llll}
0 & 1 & 0 & 0 \\
1 & 0 & 0 & 0 \\
0 & 0 & 0 & 1 \\
0 & 0 & 1 & 0
\end{array}\right], \quad L M_{J_{1}}(g)=\left[\begin{array}{llll}
0 & 0 & 0 & 1 \\
0 & 0 & 1 & 0 \\
0 & 1 & 0 & 0 \\
1 & 0 & 0 & 0
\end{array}\right] .
$$


Let $G$ be a maximal subgroup of $J_{1}$. Computing $1_{G}^{\overline{\widehat{C}}}$ by $(2.20)$ of [14] we get

$$
1_{\widehat{G}} \overline{(g)}(g)\left[\begin{array}{rrr}
-1 & 0 & 0 \\
1 & 0 & 1 \\
1 & 1 & 0
\end{array}\right]
$$

thus $\chi(1 \overline{\widehat{G}})(g)=-1$.

1.9. FACT. Let $S=J^{0} \simeq \mathscr{M}^{0}(G,\{1,2, \ldots, m\},\{1,2, \ldots, n\}, C)$ and let $\operatorname{IRR}(G)$ $=\left\{\varphi_{1}, \varphi_{2}, \ldots, \varphi_{k}\right\}$ and $\theta$ the right regular representation of $G$. Let $\mathscr{R}$ be the right regular representation of $K_{0}[S]$. Then $\mathscr{R}=\tilde{\varphi} \oplus \tilde{\varphi} \oplus \cdots \oplus \tilde{\varphi}$ ( $m$ times $)$ where $\varphi=\theta^{*}\left(R M_{J}\right)=\sum_{i=1}^{k} n_{i} \varphi_{i}^{*}\left(R M_{J}\right), n_{i}=\operatorname{deg}\left(\varphi_{i}\right), i=1,2, \ldots, k$.

Furthermore, each $\varphi_{i}^{*}\left(R M_{J}\right)$ is indecomposable and nonnull and thus a principal indecomposable representation of $K_{0}[S]$. Each principal indecomposable representation of $K_{0}[S]$ is of this form. $\varphi_{i}^{*}\left(R M_{J}\right)$ has multiplicity $n_{i} m$ in $\mathscr{R}$.

Proof. For $s=(g, i, j) \in S^{\#}$,

$$
\mathscr{R}(s)\left(\left(g_{1}, i_{1}, j_{1}\right)\right)=\left(g_{1} C\left(j_{1}, i\right) g, i_{1}, j\right)=\left(\overline{g_{1}, i_{1}, j_{1}}\right) C(\overline{g, i, j})
$$

where the bar denotes the element considered as a matrix over $G^{0}$ and the operation is matrix multiplication, and the right-hand side equals $\left(g_{1}, i_{1}, j_{1}\right) R M_{J}(s)$. Let $G=\left\{e=g_{1}, g_{2}, \ldots, g_{r}\right\}$, then with respect to the ordered basis

$$
\begin{array}{ll}
(e, 1,1), \ldots,\left(g_{r}, 1,1\right),(e, 1,2), \ldots,\left(g_{r}, 1,2\right), \ldots,\left(g_{r}, 1, n\right) \\
\quad \vdots & \ldots,\left(g_{r}, i, n\right) \\
(e, i, 1), \ldots, & \\
\quad \vdots & \ldots,\left(g_{r}, m, n\right),
\end{array}
$$

$\mathscr{R}=\tilde{\varphi} \oplus \cdots \oplus \tilde{\varphi}$. The expression $\varphi=\sum_{i=1}^{k} n_{i} \varphi_{i}^{*}\left(R M_{J}\right)$ follows from Wedderburn's theorem.

If $\varphi_{i}^{*}\left(R M_{J}\right)$ were decomposable, say $\psi_{1} \oplus \psi_{2} \oplus \cdots \oplus \psi_{l}$, then by $[2,(9.2)]$, $\varphi_{i}^{*}\left(R M_{J}\right)$ would have at least $l$ nonnull constituents. But, by Fact 2.14 of [14], $\varphi_{i}^{*}\left(R M_{J}\right)$ has a unique nonnull constituent. Thus $\varphi_{i}^{*}\left(R M_{J}\right)$ is indecomposable and hence a principal indecomposable representation.

1.10. Corollary. Let $J$ be a regular $\mathscr{J}$-class of $S^{1}, J^{0} \simeq \mathscr{M}^{0}(G, A, B, C)$ and let $\varphi \in \operatorname{IRR}(G)$, then $\varphi^{*}\left(R M_{J}\right)$ is indecomposable. In particular, the action of a right letter mapping semigroup on its distinguished $\mathscr{J}$-class is indecomposable.

Proof. $\left.\varphi^{*}\left(R M_{J}\right)\right|_{J \cup F(J)}$ is indecomposable by 1.9 , thus $\varphi^{*}\left(R M_{J}\right)$ is indecomposable.

1.11. Definition and Notation. $(X, S)$ denotes a right mapping semigroup (or right transformation semigroup), $X=D_{n}=\{1,2, \ldots, n\}, n \geqq 2$. For $i \in X, s \in S$, let $i . s=(i) s$. For $s \in S$, range $(s)=X . s=\{i . s: i \in X\}$. The rank of $s$, denoted by $r(s)$, is $|X . s|$ and $\bmod (s)$ is the partition induced by $s$, i.e., $i_{1} \equiv i_{2} \bmod (s)$ iff $i_{1} . s=i_{2} . s$. 
$\tau(s)=|\{i \in X: i . s=i\}|$ is the number of fixed points of $s$. $s$ is null $\Leftrightarrow s$ does not belong to a subgroup of $S$. $e(s)$ denotes the unique idempotent among the powers of $s$.

Clearly, $r\left(s^{k}\right) \leqq r(s)$ for all $k \geqq 1$ and $s_{1} \mathscr{J} s_{2} \Rightarrow r\left(s_{1}\right)=r\left(s_{2}\right)$, thus we may speak of the rank $r(J)$ of a $\mathscr{J}$-class $J$ or $r(H)$ of an $\mathscr{H}$-class $H$, which is defined to be the rank of any element of $J$ or $H$, respectively. Note, however, that two elements of the same rank need not be $\mathscr{J}$-equivalent. Also, $J_{1} \leqq J_{2} \Rightarrow$ for any $s_{i} \in J_{i}, i=1,2$, $r\left(s_{1}\right) \leqq r\left(s_{2}\right)$.

For $s \in S$, let $A(s)$ be the subset of $X$ of highest cardinality such that $\left.s\right|_{A(s)}$ is a permutation. $\left.s\right|_{A(s)}$ is called the permutation part of $s$ and is denoted by $\operatorname{PP}(s)$. $\mathrm{PP}(s)$ is well defined by the following lemma.

1.12. Lemma. Let $(X, S)$ be a faithful mapping semigroup and let $s \in S$, then

a. The following are equivalent:

(1) $s$ belongs to a subgroup of $S$.

(2) $r\left(s^{2}\right)=r(s)$.

(3) range $(s)$ is a set of representatives for $\bmod (s)$, i.e., each equivalence class of mod $(s)$ contains exactly one element from range $(s)$.

(4) $A(s)=$ range $(s)$.

(5) $\left.s\right|_{\text {range(s) }}$ is a permutation.

b. The following are equivalent:

(1) $s^{2}=s$.

(2) $\left.s\right|_{\text {range(s) }}$ is the identity map.

(3) $s$ is the identity map on some subset of $X$ of cardinality $r(s)$.

(4) $r(s)=\tau(s)$.

c. For $s_{1}, s_{2} \in S, s_{1} \mathscr{H} s_{2}$ implies range $\left(s_{1}\right)=$ range $\left(s_{2}\right)$.

d. Let $e(s)$ be the unique idempotent among the powers of $s$, then $A(s)=A(e(s))$ $=\operatorname{range}(e(s))$.

Proof. The proof is elementary, and will be omitted.

1.13. Definition and Notation. Throughout the remainder of the section, $(X, S)$ will denote a faithful right mapping semigroup with $|X|=n$ such that $S$ has an identity element 1 and $i .1=i$ for all $i \in X$. The maximal subgroup $U$ of $S$ containing 1 will be called the group of units of $S .(X, U)$ will denote the group of units of some mapping semigroup $(X, S)$ even when $(X, S)$ is not mentioned explicitly.

For $i \in X, C_{i}$ is the map defined by $j . C_{i}=i$ for all $j \in X . C_{i}$ is called the constant map to $i$.

The standard representation $M: S \rightarrow \mathscr{M}(K, n)$ is defined by

$$
\begin{aligned}
(M(s))_{i j} & =1 & & \text { if } i . s=j \\
& =0 & & \text { if } i . s \neq j .
\end{aligned}
$$


$V=V(M)$ is the representation module of $M$ with basis $\left\{v_{1}, \ldots, v_{n}\right\}$ so that $v_{i} s$ $=v_{j} \Leftrightarrow i . s=j$. We say $M$ is $k$-transitive iff $(X, S)$ is $k$-transitive, i.e., for any two $k$-tuples $\left(i_{1}, \ldots, i_{k}\right),\left(j_{1}, \ldots, j_{k}\right)$ of elements of $X$ such that the $i_{l}$ 's and the $j_{m}$ 's are pairwise distinct there exists $s \in S$ such that $i_{l}, s=j_{l}$ for $l=1,2, \ldots, k$.

1.14. FACT. If $S$ contains all constant maps, then $M$ is indecomposable.

Proof. $J=\operatorname{kernel}(S)=\left\{C_{i}: i \in X\right\}$ and if 1 denotes the principal character on the (one-point) maximal subgroup of $J$, then $M=1^{*}\left(R M_{J}\right)$ which is indecomposable by Corollary 1.10. Q.E.D.

1.15. Notation. Given $(X, S)$, let $G$ be a maximal subgroup of $S$. Let $s \in G$, then by Lemma $\left.1.12 s\right|_{\text {range(s) }}$ is a permutation and range $(s)$ is independent of the element $s$ chosen. Thus $(X . s, G)$ is a permutation group. Let $M_{G}$ be the standard representation of $(X . s, G)$. By Fact 1.10 of [14], $\left.M\right|_{G}=M_{G}+$ null constituents, hence $\chi\left(M_{G}\right)=\chi\left(\left.M\right|_{G}\right)$.

If $(X, S)$ is a permutation group $(X, G)$, then, as is well known, $\#\left(1_{G}, M\right)$ is equal to the number of orbits of the permutation representation $M$, and, in particular, $\#\left(1_{G}, M\right)=1$ iff $M$ is transitive [5, Theorem (32.3)]. This is generalized in Facts 1.16 and 1.18 below.

1.16. FACT. If $J=\operatorname{kernel}(S)$ and $G$ is a maximal subgroup of $J, \#\left(1_{S}, M\right)$ is equal to the number of orbits of the permutation representation $M_{G}$ of $G$. In particular, $\#\left(1_{S}, M\right)=1$ iff $M_{G}$ is a transitive permutation representation.

Proof. By the group-theoretic result quoted above, the number of orbits of $M_{G}$ equals $\#\left(1_{G}, M_{G}\right)$ and since $1_{G}=\left(1_{S}\right)_{G}, \#\left(1_{G}, M_{G}\right)=\#\left(1_{S}, M\right)$ by Fact 2.14 of [14].

1.17. FACT. Let $T \leqq S^{1}$ with $1 \in T$ and let $\varphi$ be a representation of $S$ such that $\varphi(1)=I_{n}$. Then the number of nonnull constituents of $\varphi$ is less than or equal to the number of nonnull constituents of $\left.\varphi\right|_{T}$ (counting multiplicities).

Proof. Let $\varphi_{1}, \varphi_{2}, \ldots, \varphi_{n}$ be the nonnull constituents of $\varphi$ (counting multiplicities). Now, $\left.\varphi_{k}\right|_{T}$ is not null since $1 \in T$. Thus $\left.\varphi_{k}\right|_{T}$ has at least one nonnull constituent and the inequality follows.

1.18. FACT. If $(X, U)$ is transitive, $\#\left(1_{S}, M\right)=1$.

Proof. By Fact $1.161 \leqq \#\left(1_{S}, M\right)$ and by Fact $1.17 \#\left(1_{S}, M\right) \leqq \#\left(1_{S \mid U},\left.M\right|_{U}\right)$ $=\#\left(1_{U}, M_{U}\right)$ and since $M_{U}$ is transitive this equals 1 (by the result quoted in the proof of 1.16). Hence, $\#\left(1_{S}, M\right)=1$.

1.19. Remark. If $(X, U)$ is transitive, $J=\operatorname{kernel}(S)$ and $G$ a maximal subgroup of $J$, then by 1.16 and $1.18, M_{G}$ is a transitive permutation representation. The converse is trivially false since for any mapping semigroup $S$ such that kernel $(S)$ has rank $1, \#\left(1_{s}, M\right)=1$.

1.20. Notation. Given $(X, S)$ and $s \in S$, let $\alpha_{i}(s), i=1,2, \ldots, n$, be the number of $i$-cycles in the decomposition of PP $(s)$ into disjoint cycles, thus the $\alpha_{i}$ 's are functions from $S$ into the nonnegative integers and $\alpha_{1}=\chi(M)$. 
Given $(X, S)$ and a maximal subgroup $G$ of $S$, if $G$ contains an element $s$ such that $\mathrm{PP}(s)$ is an odd permutation, we define $\chi_{G}^{A}: G \rightarrow\{1,-1\}$ by $\chi_{G}^{A}(s)=\operatorname{sgn}(\mathrm{PP}(s))$. Note that $\chi_{G}^{A}$ is defined only if $G$ contains an odd permutation. We refer to $\chi_{G}^{A}$ as the alternating character of $G$.

1.21. RemarK. As is well known, the irreducible characters of $\mathscr{S}_{k}$ are in one-toone correspondence with the ordered partitions $\left(\lambda_{1}, \ldots, \lambda_{r}\right)$ of $k$ where $\lambda_{1} \geqq \lambda_{2}$ $\geqq \cdots \geqq \lambda_{r}>0$ and $k=\sum_{i=1}^{r} \lambda_{i}$, the character corresponding to $\left(\lambda_{1}, \ldots, \lambda_{r}\right)$ is denoted by $\chi^{\left(\lambda_{1}, \ldots, \lambda_{r}\right)}$ (see e.g., Littlewood [9]). If $G_{k}$ is a maximal subgroup of $S$ of rank $k$ and $G_{k} \simeq \mathscr{S}_{k}$, we denote the character corresponding to the partition $\left(\lambda_{1}, \ldots, \lambda_{p}\right)$ of $k$ by $\chi_{k}^{\left(\lambda_{1}, \lambda_{2}, \ldots, \lambda_{p}\right)}$.

Given $\chi^{\left(\lambda_{1}, \ldots, \lambda_{r}\right)}$, the dimension of $\chi^{\left(\lambda_{1}, \lambda_{2}, \ldots, \lambda_{r}\right)}$ is defined to be $n-\lambda_{1}$ (see Frobenius [7]).

Our objective is to get information about $S$ from various assumptions about the transitivity of its group of units. We will need the following theorem due to Frobenius [7]: If a faithful permutation group $(X, G)$ is $2 k$-transitive, then for every irreducible character $\chi$ of $\mathscr{S}_{n}$ of dimension $\leqq k,\left.\chi\right|_{G}$ is irreducible.

Tsuzuku [16] has proved the converse of this theorem. Let $(X, G)$ be a faithful permutation group and let $k<n / 2$. If for every irreducible character $\chi$ of $\mathscr{S}_{n}$ of dimension $k,\left.\chi\right|_{G}$ is irreducible, then $(X, G)$ is $2 k$-transitive. (The cases $k=1,2$ have been proved by Frobenius.)

Thus in particular $(X, G)$ is 2-transitive $\Leftrightarrow \alpha_{1}-1$ is irreducible and $(X, G)$ is 4-transitive $\Leftrightarrow\left(\alpha_{1}-1\right)\left(\alpha_{1}-2\right) / 2-\alpha_{2}$ and $\alpha_{1}\left(\alpha_{1}-3\right) / 2+\alpha_{2}$ are irreducible.

If $\chi^{\left(\lambda_{1}, \ldots, \lambda_{p}\right)}$ is an irreducible character of $\mathscr{S}_{n}$ and $\left.\chi^{\left(\lambda_{1}, \ldots, \lambda_{p}\right)}\right|_{G}$ is irreducible, we will denote $\left.\chi^{\left(\lambda_{1}, \lambda_{2}, \ldots, \lambda_{p}\right)}\right|_{G}$ by $\chi^{\left(\lambda_{1}, \ldots, \lambda_{p}\right)}$. For example, given $(X, S)$ and a maximal subgroup $G$ of rank $k$, if $\chi_{G}^{A}$ is defined, then $\chi_{G}^{A}=\chi_{k}^{(1 k)}$.

1.22. FACT. Given $(X, S)$, define integer-valued functions $\mu_{k}, k=1,2, \ldots, n-1$, on $S$ as follows:

Let $\pi=\left(\lambda_{p}, \lambda_{p-1}, \ldots, \lambda_{2}, \lambda_{1}\right)$ be an ordered partition of $k ;$ i.e., $\lambda_{p} \geqq \lambda_{p-1} \geqq \cdots \geqq \lambda_{2}$ $\geqq \lambda_{1}>0$ and $\sum_{i=1}^{p} \lambda_{i}=k$. Let $a(\pi)=(-1)^{k+p}$ and let $\beta_{i}=\beta_{i}(\pi), i=1,2, \ldots, k$, be the number of $\lambda_{j}$ 's in $\pi$ such that $\lambda_{j}=i$. Then

$$
\mu_{k}=\sum_{\pi} a(\pi)\left(\begin{array}{c}
\alpha_{1} \\
\beta_{1}
\end{array}\right)\left(\begin{array}{c}
\alpha_{2} \\
\beta_{2}
\end{array}\right) \cdots\left(\begin{array}{c}
\alpha_{k} \\
\beta_{k}
\end{array}\right)
$$

where the summation is over all ordered partitions of $k$. (Thus

$$
\begin{gathered}
\mu_{1}=\alpha_{1}, \quad \mu_{2}=\alpha_{1}\left(\alpha_{1}-1\right) / 2-\alpha_{2}, \\
\left.\mu_{3}=\alpha_{1}\left(\alpha_{1}-1\right)\left(\alpha_{1}-2\right) / 6-\alpha_{1} \alpha_{2}+\alpha_{3}, \quad \text { etc. }\right)
\end{gathered}
$$

Then, $\mu_{k}$ is a character of $S$ which vanishes on all elements of rank $<k$.

Furthermore, if $J$ is a regular $\mathscr{J}$-class of $S$ of rank $k$ and $G$ is a maximal subgroup of $S$ contained in $J$, then $\left.\mu_{k}\right|_{G}=\chi_{G}^{A}$ if $G$ contains an odd permutation and otherwise $\left.\mu_{k}\right|_{G}=1_{G}$. 
Proof. Let $V=V(M)$ and let $V_{k}$ be the subspace of skew-symmetric tensors in $V \otimes k\left(V \otimes k\right.$ is the $k$ th tensor power of $V$, i.e., $\left.V^{1}=V, V \otimes k+1=(V \otimes k) \otimes V\right), 1 \leqq k<n$. (See Curtis and Reiner [5, p. 452].) $V_{k}$ has a basis consisting of elements of the form

$$
v_{i_{1}, \ldots, i_{k}}=\sum_{\sigma \in \mathscr{S}_{k}} \operatorname{sgn}(\sigma) v_{i_{\sigma(1)}} \otimes v_{i_{\sigma(2)}} \otimes \cdots \otimes v_{i_{\sigma(k)}},
$$

where $i_{1}, \ldots, i_{k} \in X$ and $i_{1}, \ldots, i_{k}$ are pairwise distinct.

Now, it is easy to see that if $s \in S$ is a permutation when restricted to $\left\{i_{1}, i_{2}, \ldots, i_{k}\right\},\left(v_{i_{1} i_{2} \cdots i_{k}}\right) s=\operatorname{sgn}\left(\left.s\right|_{\left\{i_{1}, i_{2}, \ldots, i_{k}\right\}}\right) v_{i_{1}, s, \ldots, i_{k} . s}$ and if $\left.s\right|_{\left\{i_{1}, i_{2}, \ldots, i_{k}\right\}}$ is not oneto-one, $\left(v_{i_{1}, i_{2}, \ldots, i_{k}}\right) s=0$. Thus $V_{k}$ is a $K[S]$-submodule of $V{ }^{\otimes k}$. Let $\varphi_{k}$ be the representation carried by $V_{k}$. Then $\operatorname{deg}\left(\varphi_{k}\right)=\left(\begin{array}{c}n \\ k\end{array}\right)$ and $\varphi_{k}$ vanishes on all elements of rank $<k$.

Now, by a somewhat tedious direct verification, $\mu_{k}=\chi\left(\varphi_{k}\right)$.

We now prove the second statement. For $s \in G, \sum_{i=1}^{k} i \alpha_{i}(s)=k$, thus for any partition $\pi$ of $k, \sum_{i=1}^{k} i \alpha_{i}(s)=\sum_{i=1}^{k} i \beta_{i}(\pi)$. If, for some $i, \beta_{i}(\pi)>\alpha_{i}(s)$, then

$$
\left(\begin{array}{l}
\alpha_{i}(s) \\
\beta_{i}(\pi)
\end{array}\right)=0
$$

If, for some $j, \beta_{j}(\pi)<\alpha_{j}(s)$, then by the equality above, for some $i, \beta_{i}(\pi)>\alpha_{i}(s)$ and again

$$
\left(\begin{array}{l}
\alpha_{i}(s) \\
\beta_{i}(\pi)
\end{array}\right)=0
$$

Thus exactly one term in the sum defining $\mu_{k}(s)$ is not zero, and for that term, $\alpha_{i}(s)=\beta_{i}(\pi)$ for all $i \leqq k$. Furthermore, $a(\pi)=1$ if $\mathrm{PP}(s)$ is even and $\alpha(\pi)=-1$ if PP $(s)$ is odd, where $\pi$ is the partition induced by the decomposition of PP $(s)$ into disjoint cycles. Thus, $\mu_{k}(s)=\operatorname{sgn}(\mathrm{PP}(s))$. This proves Fact 1.22.

REMARK. The subspace of symmetric tensors in $V \otimes k$ is not a $K[S]$-submodule if $S$ is not a group, hence there is no analog of Fact 1.22 for the symmetric case.

1.23. Definition. Given $(X, S)$, if $\chi$ is a character of $U$ that can be expressed in terms of the $\alpha_{i}$ 's we can extend $\chi$ to an integer-valued function $\chi_{*}$ on $S$ by using the same formal expression for $\chi$ in terms of the $\alpha_{i}$ 's. $\chi_{*}$ is not, in general, a character of $S$; in fact, even the restriction of $\chi_{*}$ to a maximal subgroup need not be a character.

The next theorem states that if the group of units is multiply transitive with a sufficiently high degree of transitivity, then for certain irreducible characters $\chi$ of $U, \chi_{*}$ is an irreducible character of $S$. It therefore provides the first nontrivial explicit formula for an irreducible character of a finite semigroup.

1.24. TheOREM. Let $(X, U)$ be $2 k$-transitive, $k \geqq 1$, and let $S$ contain an element of rank $k+1$. Then for all $i, 1<i \leqq k+1$, and all maximal subgroups $G_{i}$ of rank $i$,

$$
\left(\chi_{G_{l}}^{A}\right)^{\pi}=\chi_{*}^{\left(n-i+1,1^{1-1}\right)}=\sum_{\pi} a(\pi)\left(\begin{array}{c}
\alpha_{1}-1 \\
\beta_{1}
\end{array}\right)\left(\begin{array}{c}
\alpha_{2} \\
\beta_{2}
\end{array}\right) \cdots\left(\begin{array}{c}
\alpha_{i-1} \\
\beta_{i-1}
\end{array}\right)
$$


where the summation is over all the ordered partitions of $i-1$, and where $a(\pi)$ and $\beta_{i}=\beta_{i}(\pi)$ are as in 1.22. In particular,

$$
\operatorname{deg}\left(\left(\chi_{G_{i}}^{A}\right)^{\wedge}\right)=\left(\begin{array}{c}
n-1 \\
i-1
\end{array}\right)
$$

Furthermore, $\left.\left(\chi_{G_{i}}^{A}\right)^{ }\right|_{U}$ is irreducible.

Proof. We need the following lemma.

1.25. Lemma. Let $(X, U)$ be $m$-transitive, $1 \leqq m \leqq n-1$, then

a. If $S$ contains an element of rank $k \leqq m$, then for every $k$-subset $A$ of $X, S$ contains an idempotant whose range is $A$.

b. If $S$ contains an element of rank $k \leqq m$, then $S$ contains elements of all ranks $r \leqq k$.

c. If $S$ contains an idempotent e of rank $k \leqq m$, then $H_{e} \simeq \mathscr{S}_{k}$.

Proof. a. Let $s$ be an element of rank $k$. For all $u \in U, \bmod (s u)=\bmod (s)$ and by $m$-transitivity, there exist $u \in U$ such that range $(s u)$ is a set of representatives for $\bmod (s u)=\bmod (s)$, thus by Lemma $1.12, r(e(s u))=k$. Hence we may assume that $s$ is an idempotent. Now, by $m$-transitivity there exists $u \in U$ so that range $(s u)=A$, then $u^{-1} s u$ is the required idempotent.

b. Let $s$ be an element of rank $k$. By $m$-transitivity, there exists $u \in U$ such that $s u$ is null. Thus we may assume $s$ is null. Let range $(s)=\left\{i_{1}, i_{2}, \ldots, i_{k}\right\}$ and $\bmod (s)$ $=\left\{A_{1}, A_{2}, \ldots, A_{k}\right\}$. Since $k<n$, at least one of the $A_{i}$ 's, say $A_{1}$, has cardinality $>1$. Pick $u \in U$ such that $i_{j} . u \in A_{j}$ for $j=1,2, \ldots, k-1$ and $i_{k} \cdot u \in A_{1}$. Then $r\left((s u)^{2}\right)$ $=k-1$.

c. Without loss of generality, let range $(e)=\{1,2, \ldots, k\}=D_{k}$. By $k$-transitivity, for all $\sigma \in \mathscr{S}_{k}$, there exists $u \in U$ such that $\left.u\right|_{D_{k}}=\sigma$, thus eue $\left.\right|_{D_{k}}=\sigma$. Now, since $u$ permutes range $(e)$, i.e $=j . e$ iff $i(e u e)=j(e u e)$. Thus $\bmod (e u e)=\bmod (e)$ and clearly range $(e)=\operatorname{range}(e u e)$. Thus $(e u e) \mathscr{H} e$ in $F_{R}(X)$ and so $(e u e) \mathscr{H} e$ in $S$. Hence $H_{e} \simeq \mathscr{S}_{k}$. This proves the lemma.

1.26. Corollary (OF Lemma 1.25). If $(X, U)$ is m-transitive and $S$ contains an element of rank $k \leqq m$, then all elements of rank $k$ are $\mathscr{J}$-equivalent and the $\mathscr{J}$-class consisting of all elements of rank $k$ is regular.

Proof. Let $s$ and $t$ be regular elements of rank $k$ (these exist by Lemma 1.25). By the proof of the lemma, there exists $u \in U$ such that $\bmod (s u)=\bmod (s)$ and range $(s u)=\operatorname{range}(t)$. Thus $s \mathscr{R}(s u)$ in $F_{R}(X)$ and $(s u) \mathscr{L} t$ in $F_{R}(X)$. Hence by Proposition 6.2 of [11], $s \mathscr{R}(s u)$ and $(s u) \mathscr{L} t$ in $S$ and thus $s \mathscr{J} t$. The last assertion is clear. This proves the corollary.

We proceed with the proof of the theorem.

By Lemma $1.25, S$ contains idempotents of all ranks $i \leqq k+1$ and $G_{i} \simeq \mathscr{S}_{i}$ for $i \leqq k+1$. Furthermore, by Corollary 1.26 , there is a unique $\mathscr{J}$-class of rank $i$, for $i \leqq k+1$. 
$(X, U)$ is $2 k$-transitive and hence 2-transitive, thus $\left.\left(\alpha_{1}-1_{S}\right)\right|_{U}$ is irreducible and so, by $1.17, \alpha_{1}-1$ is irreducible. Let $J=$ apex $\left(\alpha_{1}-1\right)$, then by the $\leqq$-minimality of $J, r(J)=2$ and $J$ is in fact the unique $\mathscr{J}$-class of $S$ of rank 2. Let $G$ be a maximal subgroup of $S$ contained in $J$, then $G=G_{2} \simeq Z_{2}$ and $\left.\left(\alpha_{1}-1\right)\right|_{G_{2}}=\chi_{G_{2}}^{A}$, thus by Fact 2.14 of [14], $\left(\chi_{G_{2}}^{A}\right)^{\nwarrow} \leqq \alpha_{1}-1$, and since $\alpha_{1}-1$ is irreducible, $\left(\chi_{G}^{A}\right)^{\nwarrow}=\alpha_{1}-1$. In particular

$$
\operatorname{deg}\left(\chi_{G_{2}}^{A}\right)^{\nwarrow}=n-1=\left(\begin{array}{l}
n-1 \\
2-1
\end{array}\right)
$$

thus (1) holds for $i=1$.

We proceed by induction on $i$. Assume (1) holds for $i \leqq k$. Then $\left(\chi_{G_{i}}^{A}\right)^{\pi}$ $=\chi_{*}^{\left(n-i+1,1^{i-1)}\right.}$. Now, by Fact $1.22,\left.\mu_{i}\right|_{G_{i}}=\chi_{G_{i}}^{A}$, thus by Fact 2.14 of [14], $\left(\chi_{G_{i}}^{A}\right)^{\wedge}$ is a constituent of $\mu_{i}$ and in fact, the only constituent with apex of rank $i$. Furthermore, $\left.\mu_{i}\right|_{U}=\chi^{\left(n-i+1,1^{i-1}\right)}+\chi^{\left(n-i, 1^{i}\right)}$. This follows from the formula for $\chi^{\left(n-i, 1^{i}\right)}$ in terms of the $\alpha_{i}$ 's which can be proved using the method described in Murnaghan [10, pp. 143-147]. Further, by Frobenius' theorem quoted above, since both characters on the right-hand side have dimension $\leqq k$, they are irreducible. Thus by Fact $1.17, \mu_{i}$ has at most two constituents. Further, since $\left(\chi_{G_{i}}^{A}\right)^{\nwarrow}$ is a constituent and by the induction hypothesis

$$
\operatorname{deg}\left(\left(\chi_{G_{i}}^{A}\right)^{\nwarrow}\right)=\left(\begin{array}{c}
n-1 \\
i-1
\end{array}\right)<\left(\begin{array}{l}
n \\
i
\end{array}\right)=\operatorname{deg} \mu_{i}
$$

it has exactly two constituents, the other constituent $\varphi$ having degree

$$
\left(\begin{array}{l}
n \\
i
\end{array}\right)-\left(\begin{array}{c}
n-1 \\
i-1
\end{array}\right)=\left(\begin{array}{c}
n-1 \\
i
\end{array}\right)
$$

and apex $J$ of rank $>i$.

To complete the proof we need the following lemmas:

1.27. Lemma. Let $(X, G)$ be 2-transitive. Define the integer-valued function $\eta$ on $G$ by $\eta(\sigma)=\operatorname{sgn}(\sigma) \alpha_{1}(\sigma)$. Then $\eta=\operatorname{sgn}(\cdot)+\chi$ where $\chi$ is irreducible.

If $(X, G)=\left(X, \mathscr{S}_{n}\right)$, then $\eta=\chi^{\left(1^{n}\right)}+\chi^{\left(2,1^{n-1}\right)}$.

Proof. $\alpha_{1}-1_{G}$ is an irreducible character, thus $\chi=\operatorname{sgn}(\cdot)\left[\alpha_{1}-1_{G}\right]$ is an irreducible character. Now,

$$
\eta=\operatorname{sgn}(\cdot) \alpha_{1}=\operatorname{sgn}(\cdot)+\operatorname{sgn}(\cdot)\left[\alpha_{1}-1\right]=\operatorname{sgn}(\cdot)+\chi .
$$

If $(X, G)=\left(X, \mathscr{S}_{n}\right), \operatorname{sgn}(\cdot)=\chi^{\left(1^{n}\right)}$ and $\alpha_{1}-1=\chi^{(n-1,1)}$. Further, if $\mu$ is a partition and $\lambda$ is the partition conjugate to $\mu, \chi^{\left(1^{n}\right)} \cdot \chi^{(\mu)}=\chi^{(\lambda)}$ (see Littlewood [9, p. 71]), thus $\chi=\chi^{\left(2,1^{n-1}\right)}$.

1.28. Corollary. Given $(X, S)$, if $J$ is a $\mathscr{J}$-class of $S$ of rank $k+1$ and $G_{k+1}$ $=G_{J} \simeq \mathscr{S}_{k+1}$, then

$$
\left(\mu_{k}\right)_{G_{k+1}}=\chi_{k+1}^{\left(2,1^{k-1)}\right.}+\chi_{k+1}^{\left(1^{k+1}\right)}
$$


Proof. Since the character $\mu_{k}$ depends only on the permutation parts of the mappings involved, we may consider $(X . e, e S e)$ instead of $(X, S)$, where $e$ is the identity of $G_{k+1}$. Thus we may assume, without loss of generality, that $k=n-1$ and so $G_{k+1}=U \simeq \mathscr{S}_{n}$. Now, in computing the character $\left.\mu_{n-1}\right|_{U}$ we may replace the standard action of $U$ on $V_{n-1}$ by the action of $U$ on $V_{n-1}$ by $v_{i} . u=\operatorname{sgn}(u) v_{i . u}$ (since if $\bar{m}=X-\{m\},\left(v_{\bar{m}}\right) u=\lambda v_{\bar{m}} \Leftrightarrow m . u=m$ and $\lambda=\operatorname{sgn}\left(\left.u\right|_{\bar{m}}\right)=\operatorname{sgn}(u)$ ). Thus the corollary follows from Lemma 1.27 .

1.29. LemMA. Given $(X, S)$, if $U$ contains an odd permutation and

$$
e=\frac{1}{n !} \sum_{u \in U} \operatorname{sgn}(u) u,
$$

then

a. For all $s \in S-U$, es $=0$.

b. If $\varphi$ is an irreducible representation of $S$ with apex $J \neq U, \chi\left(\left.\varphi\right|_{U}\right)$ does not contain $\chi_{n}^{\left(1^{n}\right)}$ as a constituent.

Proof. If $s \notin U, r(s)<n$, thus there exists an equivalence class $A$ of $\bmod (s)$ of cardinality $\geqq 2$. Let $i, j \in A, i \neq j$. Then $\sigma \rightarrow \sigma^{\prime}=\sigma(i j)$ is a bijection of the set of all even permutations in $U$ onto the set of all odd permutations. Thus it is easy to see that $\sigma s=\sigma^{\prime} s$. Thus in the product es, the terms with the plus sign cancel the terms with the minus sign, so $e s=0$.

To prove b, note that $e K[U]$ is the representation module of $\chi^{\left(1^{n}\right)}$. Now, by a, $e K[S]=e K[U]$, and by Lemma 1.28 of $[14], e K[U]=(e K[U])^{S}$. Thus $\left(\chi_{n}^{\left(1^{n}\right)}\right)^{S}$ $=\chi_{n}^{\left(1^{n}\right)}$ and so, for every irreducible representation $\varphi$ of $S$ with apex $J \neq U$, $\#\left(\varphi,\left(\chi_{n}^{(1 n)}\right)^{S}\right)=0$. Hence by the generalized Frobenius reciprocity theorem, [14, (1.29)], since $K[U]$ is semisimple, $\#\left(\chi^{\left(1^{n}\right)},\left.\varphi\right|_{U}\right)=0$. This proves $\mathrm{b}$ and thus Lemma 1.29 .

1.30. Lemma. Under the hypothesis of Theorem 1.24, if $J_{k}$ denotes the $\mathscr{J}$-class of $S$ consisting of the elements of rank $k$, then $\chi_{G_{k}}^{A^{*}}\left(R M_{J_{k}}\right)=\varphi_{k}$, where $\varphi_{k}$ is defined in the proof of 1.22 .

Proof. By Lemma 1.25 , for any $k$-subset $A$ of $X, J_{k}$ contains an element whose range equals $A$. Now, if $s, t \in S \leqq F_{R}(X)$, by Proposition 6.2 of [11], $s \mathscr{L} t$ in $S \Leftrightarrow s \mathscr{L} t$ in $F_{R}(X) \Leftrightarrow$ range $(s)=$ range $(t)$. Thus the $\mathscr{L}$-classes of $J_{k}$ are in oneto-one correspondence with the $k$-subsets of $X$. Hence

$$
\operatorname{deg}\left(\chi_{G_{k}}^{A^{*}}\left(R M_{J_{k}}\right)\right)=\left(\begin{array}{l}
n \\
k
\end{array}\right)=\operatorname{deg}\left(\varphi_{k}\right)
$$

Further, by the definition of $V_{k}$ (see the proof of Fact 1.22), for every element $s$ of rank $\geqq k, \varphi_{k}(s)=\chi_{G_{k}}^{A^{*}}\left(R M_{J_{k}}\right)(s)$ and for every element $s$ of rank $<k, \varphi_{k}(s)$ $=\chi_{G_{k}}^{A_{k}^{*}}\left(R M_{J_{k}}\right)(s)=0$. This proves the lemma. 
1.31. Lemma. Let $J$ be a regular $\mathscr{J}$-class of $S, J^{0} \simeq \mathscr{M}^{0}(G, A, B, C)$. Let $\varphi \in \operatorname{IRR}(G)$ and suppose the rows of $\varphi^{*}(C)$ are linearly independent. Let $e=e^{2} \in S$ such that $J<J_{e}$, then $\mathrm{J} e$ is a regular $\mathscr{J}$-class of $e S e$ and if $C^{\prime}$ is the structure matrix of $e J e$, then the rows of $C^{\prime}$ are linearly independent.

Proof. It is easy to verify that $e J e$ is a regular $\mathscr{J}$-class. Without loss of generality assume that $R M_{J}(e)$ fixes the first $i$ rows and $L M_{J}(e)$ fixes the first $j$ columns of $C$. Then

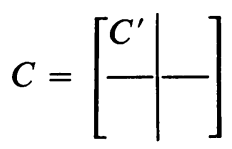

where $C^{\prime}$ is $i \times j$. Thus, if $\operatorname{deg}(\varphi)=m, \varphi^{*}\left(C^{\prime}\right)$ is the $m i \times m j$ submatrix at the upperleft corner of $\varphi^{*}(C)$.

Now, by hypothesis, the first $m i$ rows of $\varphi^{*}(C)$ are linearly independent, thus the first $m i$ rows of $\left[\varphi^{*}\left(R M_{J}\right)(e)\right] \varphi^{*}(C)$ are linearly independent. Thus by the linked equation $\left(7.2 .14(\mathrm{~d})\right.$ of [8]) the first $m i$ rows of $\varphi^{*}(C)\left[\varphi^{*}\left(L M_{J}\right)(e)\right]$ are linearly independent. But the last $m|A|-m j$ columns of $\varphi^{*}(C)\left[\varphi^{*}\left(L M_{J}\right)(e)\right]$ are multiples of the first $m j$ columns, thus it follows easily that the rows of $\varphi\left(C^{\prime}\right)$ are linearly independent. Q.E.D.

We can now complete the proof of the theorem. We claim that $\left(\chi_{G_{i}}^{A}\right) \overline{\widehat{G}}_{G_{1+1}}=\chi^{\left(2,1^{1-1}\right)}$. We know that $\left(\chi_{G_{i}}^{A}\right)_{G_{i+1}}^{\bar{G}_{i+1}} \leqq\left(\mu_{i}\right)_{G_{i+1}}$ and, by Corollary 1.28, $\left(\mu_{i}\right)_{G_{i+1}}=\chi_{i+1}^{\left(2,1^{i-1}\right)}+\chi_{i+1}^{\left(1^{i+1}\right)}$. $\left.\left(\chi_{G_{i}}^{A}\right) \bar{\approx}\right|_{G_{i+1}}$ is not null. Assume $\left(\chi_{G_{i}}^{A}\right) \overline{\hat{G}}_{G_{i+1}}=\chi_{i+1}^{(1+1)}$, then if $e$ is the identity element of $G_{i+1}$, then, in $e S e$, the representation determined by the alternating character on a maximal subgroup $G$ of $e J_{i} e$ is one dimensional. Thus if $C$ is the structure matrix of $e J_{i} e, C^{\prime}=\chi_{G}^{A^{*}}(C)$ has row-rank 1. But this is clearly impossible. (By permuting rows and columns if necessary, assume $C^{\prime}(1,1) \neq 0$. Then since $G_{i+1} \simeq \mathscr{S}_{i+1}$, and since the $\mathscr{L}$-classes are coded by $i$-subsets of $X$, as in 1.30 , there exists $i$ such that $C^{\prime}(i, 1)=0$. Further, since $e J_{k+1} e$ is regular, the $i$ th row of $C^{\prime}$ is not identically zero. Thus the first and the last rows of $C^{\prime}$ are linearly independent.) Thus, if the claim is false, $\left(\chi_{G_{i}}^{A}\right) \hat{G}_{G_{i+1}}=\chi^{\left(2,1^{1-1}\right)}+\chi^{\left(1^{1+1}\right)}=\left(\mu_{i}\right)_{G_{i+1}}$ and by Lemma 1.30 this equals $\chi_{G_{i}}^{A^{*}}\left(R M_{J_{i}}\right)$. Hence the rows of $\chi_{G_{t}}^{A^{*}}\left(C_{J_{i}}\right)$ are linearly independent. Thus by Lemma 1.31 , the rows of $\chi_{G}^{A}(C)$ are linearly independent. Hence, in $e S e,\left(\chi_{G}^{A}\right)^{\nwarrow}=\chi_{G}^{A^{*}}\left(R M_{e J_{i} e}\right)$ $=\varphi_{i}^{i+1}$, where $\varphi_{i}^{i+1}$ is the representation defined in the proof of 1.22, with $|X|=n$ $=i+1$. Thus $\left(\chi_{G}^{A}\right)_{G} \bar{G}=\chi_{i+1}^{\left(2,1^{i-1}\right.}+\chi_{i+1}^{\left(1^{i+1}\right)}$. But by Lemma 1.29 applied to $e S e, \chi_{i+1}^{\left(1^{i+1}\right)}$ is not a constituent of $\left(\chi_{G}^{A}\right)_{G_{i+1}}$. Hence, our assumption is wrong and the claim is proved.

Thus $(\varphi)_{G_{i+1}}=\chi_{i+1}^{\left(1^{1+1}\right)}$. Now, $\varphi$ is irreducible with apex of rank $>i$; thus apex $(\varphi)$ $=J_{i+1}$ and $\varphi^{2}=\chi_{G_{i+1}}^{A}$ and so $\varphi=\left(\chi_{G_{i+1}}^{A}\right)^{\pi}$. Hence $\mu_{i}=\left(\chi_{G_{i}}^{A}\right)^{\pi}+\left(\chi_{G_{i+1}}\right)^{\nwarrow}$.

Finally, since $\left.\mu_{i}\right|_{U}=\left.\left(\chi_{G_{i}}^{A}\right)^{\wedge}\right|_{U}+\left.\varphi\right|_{U},\left.\left(\chi_{G_{i+1}^{A}}^{A}\right)^{\wedge}\right|_{U}=\chi^{\left(n-i, 1^{i}\right)}$. Now, $\mu_{i}$ and $\left(\chi_{G_{i}}^{A}\right) \nwarrow$ are defined by the same formulas in $S$ as in $U$, thus $\left(\chi_{G_{i+1}}^{A}\right) \overline{=}=\chi_{*}^{\left(n-i, 1^{1}\right)}$. This proves (1). The last assertion of 1.24 follows from Frobenius' theorem. 
1.32. Corollary. If $(X, U)=\left(X, \mathscr{S}_{n}\right)$ and $S$ contains an element of rank $j<n$, then (1) holds for all $i \leqq j$. In particular, if $(X, S)=\left(X, F_{R}(X)\right)$, (1) holds for all $i$.

Proof. Since $(X, U)=\left(X, \mathscr{S}_{n}\right), \chi^{\left(n-i+1,1^{i-1}\right)}$ is irreducible for all $i \leqq n$; thus the proof of (1) goes through for all $i \leqq n$.

We note that the partitions corresponding to $\left(\chi_{G_{k}}^{A}\right)^{\wedge}$ and $\left(\chi_{G_{n-k+1}}^{A}\right)^{\wedge}$ are conjugate. This indicates that there might be some kind of duality between characters whose apexes have rank $k$ and those whose apexes have rank $n-k+1$.

1.33. FACT. If $(X, U)=\left(X, \mathscr{S}_{n}\right)$, then $S$ has no irreducible representation of degree $r, 1<r<n-1$. In particular $F_{R}(X)$ has no irreducible representation of degree $r$, $1<r<n-1$.

Proof. For $n \leqq 4$, the result follows by direct verification, so assume $n>4$. Let $\varphi$ be an irreducible representation of $S$ of degree $r, 1<r<n-1$, and let $J=\operatorname{apex}(\varphi)$ and $G$ a maximal subgroup of $S$ contained in $J$. Then $\left.\varphi\right|_{U}$ is a representation of $\mathscr{S}_{n}$ of degree $r$. But, for $n>4, \mathscr{S}_{n}$ has no irreducible representations of degree $r, 1<r$ $<n-1$ (see [3, p. 466]), and only two irreducible representations of degree 1 , namely 1 and $\chi^{\left(1^{n}\right)}$. Thus $\left.\varphi\right|_{U}$ is a direct sum of copies of 1 and $\chi^{\left(1^{n}\right)}$ and so kernel $\left(\left.\varphi\right|_{U}\right) \supseteq A_{n}$.

Now, by Lemma 1.25 and Corollary 1.26 , the $\mathscr{L}$-classes of $J$ are coded by the subsets of $X$ having cardinality $k=r(J)$ and the structure matrix $C$ of $J$ contains a $2 \times 2$ submatrix of the form $\left(\begin{array}{ll}x & 0 \\ y & z\end{array}\right)$ where $x \neq 0$. Let $C_{i}, C_{j}$ be the columns of $C$ containing $\left(\begin{array}{l}x \\ y\end{array}\right)$ and $\left(\begin{array}{l}0 \\ z\end{array}\right)$ respectively, then the columns of $\left(\varphi^{\vee}\right)^{*}\left(C_{i} C_{j}\right)\left(C_{i} C_{j}\right.$ is the $m \times 2$ matrix whose columns are $C_{i}$ and $C_{j}$ ) are linearly independent. Now, $A_{n}$ acts transitively on the set of subsets of $X$ of cardinality $k$, thus there is $u \in A_{n}$ such that $R M_{J}(u) C_{i}=C_{j}$. Hence restricted to the submodule of $W$ generated by the columns of $\left(\varphi^{\vee}\right)^{*}\left(C_{i}\right)$ and $\left(\varphi^{\vee}\right)^{*}\left(C_{j}\right), u$ does not act as the identity (notation as in $[14,(2.20)])$. Thus $u \notin \operatorname{kernel}\left(\left.\varphi\right|_{U}\right)$ which is a contradiction. Hence $S$ has no irreducible representation of degree $r, 1<r<n-1$.

1.34. FACT. Given $(X, S)$, let $\varphi$ be the representation carried by the submodule $W=\left\{v_{1}-v_{i} ; i=2,3, \ldots, n\right\}$ of $V=V(M)$. Then $\varphi$ is a $\gamma$-homomorphism (i.e. one-to-one when restricted to each subgroup of $S$ ) and $\chi(\varphi)=\alpha_{1}-1$. If, moreover, $(X, U)$ is 2-transitive, then $\varphi$ is irreducible and if $J=\operatorname{apex}(\varphi)$ and $G=G_{J}$, then $M_{G}$ is 2-transitive.

Proof. $W$ is clearly a submodule and by an elementary computation, $\chi(\varphi)=\alpha_{1}-1$. Let $H$ be a subgroup of $S$, then $\left.M\right|_{H}$ is faithful and thus $M_{H}$ is faithful. But $M_{H}$ is completely reducible, hence $M_{H}=\varphi_{H} \oplus 1_{H}$. Thus $\varphi_{H}$ and also $\left.\varphi\right|_{H}$ is faithful and so $\varphi$ is a $\gamma$-homomorphism.

If $(X, U)$ is 2-transitive, then by the proof of Theorem $1.24, \alpha_{1}-1$ is irreducible. Furthermore, $\varphi_{G}=\varphi^{\curvearrowright}$ is irreducible, $M_{G}$ is a permutation representation, and $M_{G}=\varphi^{\vee} \oplus 1_{G}$. Hence, by the converse of Frobenius' theorem, $M_{G}$ is 2transitive. 
1.35. Remark. By a result of Rhodes, if $(X, U)$ is 2-transitive and $S \neq U, S$ contains all constant maps. Hence by Fact 1.14, $M$ is indecomposable. Thus, all we can hope for are results about the reduction of $M$.

1.36. COROLlaRY. If $S$ contains all maps of rank 2 , then $\alpha_{1}-1$ is irreducible.

Proof. The set of all maps of rank 2 is a $\mathscr{J}$-class $J_{2}$ of $S$ and it is easy to see that $S$ contains all constant maps and thus $\operatorname{kernel}(S)$ is the set of all constant maps. Construct $\varphi$ as in the proof of Fact 1.34. Then $\left.\varphi\right|_{\text {kernel(S) }} \equiv 0, J_{2}$ is the unique minimal $\mathscr{J}$-class of $S$-kernel $(S)$ and $G_{J_{2}}=G_{2} \simeq Z_{2}$. Furthermore, $\left(\alpha_{1}-1\right)_{G_{2}}=\left(\chi_{G_{2}}^{A}\right)$. Hence $\left(\chi_{G}^{A}\right)^{\wedge} \leqq \varphi$. Now, $S \leqq F_{R}(X)$ and the apex of the representation $\left(\chi_{2}^{\left(1^{2}\right)}\right)^{\wedge}$ of $F_{R}(X)$ is contained in $S$. Furthermore, the degree of an irreducible representation depends only on its apex and $\left(\chi_{G_{2}}^{A}\right)^{\wedge}=\left.\left(\chi_{2}^{\left(1^{2}\right)}\right)^{\wedge}\right|_{s}$. Thus $\operatorname{deg}\left(\chi_{G_{2}}^{A}\right)^{\wedge}=\operatorname{deg}\left(\chi_{2}^{\left(1^{2}\right)}\right)^{\wedge}$ $=n-1$ by Theorem 1.24. So $\operatorname{deg}\left(\chi_{G_{2}}^{A}\right)^{\wedge}=\operatorname{deg}(\varphi)$ and consequently $\left(\chi_{G_{2}}^{A}\right)^{\wedge}=\varphi$ and the result follows by Fact 1.34 .

The conclusion of Corollary 1.36 holds if $S$ contains "sufficiently many" elements of rank 2. Making this statement precise is rather cumbersome and we leave it as an exercise to the reader.

1.37. Definition. Given $(X, S)$ let the spectrum of $S, \operatorname{Spec}(S)=\{r$ : there exists $s \in S$ such that $r(s)=r<n\}$.

By Lemma 1.25, if $(X, U)$ is $k$-transitive and $S$ contains an element of rank $m$, $m \leqq k$, then $\{1,2, \ldots, m\} \subseteq \operatorname{Spec}(S)$. It seems very difficult to get information about the spectrum under weaker assumptions about the group of units. One result we have is that if $(X, U)$ is 2-primitive, then $2 \in \operatorname{Spec}(S)$. We proceed to prove this.

We need the following result on primitive permutation groups (see Wielandt [16, Theorem (8.1)]). Let $(X, G)$ be a primitive permutation group and let $\varnothing \subset Y \subset X$. Then for any two distinct $i, j \in X$, there exists $g \in G$ such that $i . g \in Y$ and $j . g \notin Y$.

1.38. Lemma. If $(X, U)$ is primitive and $s$ is a null element of rank $k \geqq 2$, then there exists $u \in U$ such that $r\left((s u)^{2}\right)>1$. Thus any $\mathscr{J}$-class of minimal rank $>1$ is regular.

Proof. If $r\left(s^{2}\right)>1$, we are done. Otherwise, let range $(s)=\left\{i_{1}, i_{2}, \ldots, i_{k}\right\}$ and $\bmod (s)=\left\{A_{1}, A_{2}, \ldots, A_{k}\right\}$. By the result quoted above, there exists $u \in U$ such that $i_{1} . u \in A_{1}$ and $i_{2} . u \notin A_{1}$. Thus not the whole range of $s u$ belongs to a single equivalence class of $\bmod (s u)=\bmod (s)$, and so $r\left((s u)^{2}\right)>1$. The last assertion follows by Lemma 1.12.

1.39. FACT. Let $(X, U)$ be 2-primitive and let $S$ contain an element of rank $k$, $1<k<n$, then $S$ contains an element of rank 2 .

Proof. Let $s$ be an element of minimal rank $r>1$. If $r=2$, we are done. Otherwise, by Lemma 1.38 (or, alternatively, by Fact 1.34), we may assume that $s$ is an idempotent. Let range $(s)=\left\{i_{1}, i_{2}, \ldots, i_{r}\right\}$, and $\bmod (s)=\left\{A_{1}, A_{2}, \ldots, A_{r}\right\}$, $i_{j} \in A_{j}, j=1,2, \ldots, r$. By hypothesis, $U_{i_{1}}$ is primitive, thus by the result quoted 
above there exists $u \in U$ such that $i_{1} \cdot u=i_{1} \in A_{1}, i_{2} \cdot u \in A_{1}$ and $i_{3} . u \notin A_{1}$. Then $1<r\left((s u)^{2}\right)<r$, contradicting the minimality of $r$. This proves Fact 1.39.

The conclusion of 1.39 does not hold under the weaker hypothesis that $(X, U)$ is 2-transitive. In fact the "gap" between the minimal rank $r, r \in \operatorname{Spec}(S)-\{1\}$ and 1 may be arbitrarily large as is shown by the following construction, based on an idea of $\mathrm{N}$. Ito.

1.40. FACT. For every prime $p$, there exists a mapping semigroup $(X, S)$ with $|X|=p^{2},(X, U)$ 2-transitive and $\operatorname{Spec}(S)=\{1, p\}$.

Proof. Let $F=\mathrm{GF}\left(p^{2}\right)=Z_{p}(\eta)$ where $\eta$ is a primitive element. Let $X=F$ and $U$ the group of affine transformations on $F$ (i.e., transformation of the form $x \rightarrow a x+b, a, b \in F, a \neq 0)$. ( $X, U)$ is 2-transitive. Let $s$ be a mapping of $X$ into itself such that range $(s)=\{0,1,2, \ldots, p-1\}$ and $\bmod (s)=\left\{A_{0}, A_{1}, \ldots, A_{p-1}\right\}$ with $A_{i}=\{i, i+\eta, i+2 \eta, \ldots, i+(p-1) \eta\}, i=0,1, \ldots, p-1$. Note that any power of $s$ will have the same range and same partition. Let $S=\langle s, U\rangle$. Now, the sets range $(s)$, $A_{i}, i=0,1, \ldots, p-1$, have the property that if $x, y$ are any two elements of the set and $y-x=d$, then the set equals $\{x, x+d, x+2 d, \ldots, x+(p-1) d\}$. Further, each element of $U$, being an affine transformation, maps arithmetic progressions in $F$ into arithmetic progressions in $F$. Thus for any $u_{1}, u_{2} \in U$ and any $k>0$ range $\left(u_{1} s^{k} u_{2}\right)$ and the blocks of $\bmod \left(u_{1} s u_{2}\right)$ have the above-mentioned property. Further, any element of $S$ is a product of elements of the form $u_{1} s^{k} u_{2}$, and all elements of this form have rank $p$. Let $t_{1}, t_{2}$ be two elements of the above form. If range $\left(t_{1}\right)$ is a set of representatives for $\bmod \left(t_{2}\right)$ then $r\left(\left(t_{1} t_{2}\right)^{2}\right)=p$, otherwise, there are distinct elements $x, y \in \operatorname{range}\left(t_{1}\right)$ such that $x, y \in B$ where $B \in \bmod \left(t_{2}\right)$. But $\mid$ range $\left(t_{1}\right)|=| B \mid=p$ and both range $\left(t_{1}\right)$ and $B$ have the property that any two elements can be extended to an arithmetic progression exhausting the set. Thus range $\left(t_{1}\right)=B$ and consequently $r\left(\left(t_{1} t_{2}\right)^{2}\right)=1$. Thus for every element $t$ of $S$ of rank $>1$, either $t$ belongs to a maximal subgroup of rank $p$ or else $t$ is null and $r\left(t^{2}\right)$ $=1$. This proves Fact 1.40 .

Using Fact 1.39 we can get a sharper form of Theorem 1.24 for $k=2$ as follows:

1.41. THEOREM. Let $(X, U)$ be 4-transitive, then $\chi=\left(\alpha_{1}-1\right)\left(\alpha_{1}-2\right) / 2-\alpha_{2}$ is an irreducible character of $S$. Further, if $J=\operatorname{apex}(\chi)$ and $G=G_{J}$ then $M_{G}$ is 3transitive.

Proof. $\chi$ vanishes for elements of rank $\leqq 2$. Thus if $S$ does not contain an element of rank $r, 2<r<n$, then $\chi=\chi_{U}^{(n-2,1,1)}$ which is irreducible by Frobenius' theorem quoted above. Let $S$ contain an element of rank $r, 2<r<n$, then by Fact 1.39, $S$ contains an element of rank 2 , and if $G_{2}$ is a maximal subgroup of rank 2 , $G_{2} \simeq Z_{2}$. Now, by the proof of Theorem 1.24, $\left(\chi_{G_{2}}^{A}\right)^{\wedge}=\alpha_{1}-1 \leqq \mu_{2}$, and $\mu_{2}$ has two constituents. Let $\varphi$ be the other constituent then

$$
\chi(\varphi)=\alpha_{1}\left(\alpha_{1}-1\right) / 2-\alpha_{2}-\left(\alpha_{1}-1\right)=\left(\alpha_{1}-1\right)\left(\alpha_{1}-2\right) / 2-\alpha_{2} .
$$


Let $k=r(\operatorname{apex}(\varphi))$. If $k=3$ or 4 , then $G$ is the corresponding symmetric group by 1.25 . Thus, assume $k \geqq 5$, then

$$
\chi\left(\varphi^{`}\right)=\left(\alpha_{1}-1\right)\left(\alpha_{1}-2\right) / 2-\alpha_{2} .
$$

Hence by Theorem 3 of Tsuzuku [16], $M_{G}$ is 3-transitive. Q.E.D.

\section{REFERENCES}

1. Dennis Allen, Jr., Some relationships between local and global structure of finite semigroups, Ph.D. Thesis, University of California, Berkeley, 1968.

2. E. Artin, C. J. Nesbitt and R. M. Thrall, Rings with minimum condition, Univ. of Michigan Publ. Math., no. 1, Univ. of Michigan Press, Ann Arbor, Mich., 1944. MR 6, 33.

3. W. Burnside, Theory of groups of finite order, Dover, New York, 1950.

4. A. H. Clifford and G. B. Preston, The algebraic theory of semigroups. Vol. 1, Math. Surveys, no. 7, Amer. Math. Soc., Providence, R. I., 1961. MR 24 \#A2627.

5. C. W. Curtis and I. Reiner, Representation theory of finite groups and associative algebras, Pure and Appl. Math., vol. XI, Interscience, New York, 1962. MR 26 \#2519.

6. W. Feit, Characters of finite groups, Benjamin, New York, 1967. MR 36 \#2715.

7. G. Frobenius, Uber die Charaktere der mehrfach transitiven Gruppen, Sitzungsbericht Preuss. Akad. Berlin 1904, 558-571.

8. Kenneth Krohn, John Rhodes and Bret Tilson, Algebraic theory of machines, languages and semigroups (M. A. Arbib, editor), Academic Press, New York, 1968, chaps. 1, 7, 8. MR 38 \#1198.

9. Dudley E. Littlewood, The theory of group characters and matrix representations of groups, Oxford Univ. Press, New York, 1940. MR 2, 3.

10. F. D. Murnaghan, Theory of representations of groups, Johns Hopkins Press, Baltimore, Md., 1938; reprint, Dover, New York, 1963. MR 31 \#258.

11. John Rhodes, Some results on finite semigroups, J. Algebra 4 (1966), 471-504. MR 34 \#1428.

12. - Characters and complexity of finite semigroups, J. Combinatorial Theory 6 (1969), 67-85. MR 38 \#4590.

13. John Rhodes and Bret Tilson, Lower bounds for complexity of finite semigroups, J. Pure Appl. Algebra 1 (1971), 79-95.

14. John Rhodes and $Y$. Zalcstein, Elementary representation and character theory of finite semigroups and its applications, Advances in Math. (to appear).

15. Bret Tilson, Complexity of two $\mathscr{J}$-class semigroups, Advances in Math. (to appear).

16. T. Tsuzuku, On multiple transitivity of permutation groups, Nagoya Math. J. 18 (1961), 93-109. MR 23 \#A1732.

17. H. Wielandt, Finite permutation groups, Academic Press, New York, 1964. MR 32 \#1252.

18. Y. Zalcstein, Complexity and character theory of finite semigroups, Ph.D. Thesis, University of California, Berkeley, 1968.

19. - On the group-complexity of finite semigroups, Advances in Math. (to appear).

University of California, Berkeley, California 94720

Stanford University, Stanford, California 94305

Current address: Datalogy Institute, University of Copenhagen, Copenhagen, Denmark 